\title{
Prevalence and severity of renal dysfunction among 1062 heart transplant patients according to criteria based on serum creatinine and estimated glomerular filtration rate: results from the CAPRI study
}

\author{
María G. Crespo-Leiro, Juan F. Delgado, María J. Paniagua, José A. Vázquez de Prada, Juan \\ Fernández-Yáñez, Luis Almenar, Beatriz Díaz-Molina, Eulalia Roig, José M. Arizón, Luis \\ Alonso-Pulpón, Iris P. Garrido, María Luisa Sanz, Luis de la Fuente, Sonia Mirabet, Nicolás \\ Manito and Javier Muñiz
}

\begin{abstract}
:
Chronic kidney disease (CKD) is staged on the basis of glomerular filtration rate; generally, the MDRD study estimate, eGFR, is used. Renal dysfunction (RD) in heart transplant (HT) patients is often evaluated solely in terms of serum creatinine (SCr). In a cross-sectional, 14-center study of 1062 stable adult HT patients aged $59.1 \pm 12.5 \mathrm{yr}$ $(82.3 \%$ men), RD was graded as absent-or-mild (AoM), moderate, or severe (this last including dialysis and kidney graft) by two classifications: SCr-RD (SCr cutoffs 1.6 and $2.5 \mathrm{mg} / \mathrm{dL}$ ) and eGFR-RD (eGFR cutoffs 60 and $30 \mathrm{~mL} / \mathrm{min} / 1.73 \mathrm{~m}^{2}$ ). SCr-RD was AoM in $68.5 \%$ of patients, moderate in $24.9 \%$, and severe in $6.7 \%$; eGFR-RD, AoM in $38.6 \%$, moderate in 52.2\%, severe in 9.2\%. Among patients evaluated <2.7, 2.7-6.2, 6.2-9.5 and $>9.5 \mathrm{yr}$ postHT (the periods defined by time-since-transplant quartiles), AoM/moderate/severe RD prevalences were $<2.7, \mathrm{SCr}-$ RD 74/21/5\%, eGFR-RD 47/47/6\%; 2.7-6.2, SCr-RD 73/22/5\%, eGFR-RD 37/56/7\%; 6.2-9.5, SCr-RD 69/24/7\%, eGFR-RD 37/54/9\%; >9.5, SCr-RD 58/32/10\%, eGFR-RD 32/52/16\%. The prevalence of severe RD increases with time since transplant. If the usual CKD stages are appropriate for HT patients, the need for less nephrotoxic immunosuppressants and other renoprotective measures is greater than is suggested by direct SCr-based grading, which should be abandoned as excessively insensitive.
\end{abstract}

The life expectancy of patients who undergo heart transplantation (HT) is steadily increasing. For the period between January 1982 and June 2006, the estimated median survival time among patients surviving the first yr after transplantation is $13 \mathrm{yr}$ (1). Longer survival brings with it a greater risk of noncardiac morbidities because of normal aging (such as diabetes), to immunosuppression (such as neoplasia and infections), and to adverse side effects of immunosuppressive therapy that do not derive directly from immunosuppression per se. In particular, nephrotoxic effects have been of concern virtually since the introduction of cyclosporine (2). If severe renal dysfunction (RD) is indicated by a serum creatinine ( $\mathrm{SCr}$ ) level $>2.5 \mathrm{mg} / \mathrm{dL}$ or end-stage renal disease (ESRD, i.e., dialysis or kidney allograft), then the current ISHLT Registry-based Kaplan-Meier estimate of the intrinsic probability of an HT patient developing severe RD is about $8 \%$ given one-yr survival and increases at almost $3 \%$ per yr thereafter (1).

Other large studies have defined severe renal dysfunction in accordance with the guidelines published in 2002 by the US National Kidney Foundation's Kidney Disease Outcomes Quality Initiative (K/DOQI) (3). These guidelines define stages of chronic kidney disease fundamentally on the basis of glomerular filtration rate (GFR) and recommend, as a convenient practical means of estimating GFR, the use of one or another of the equations developed by the Modification of Diet in Renal Disease (MDRD) Study Group (4). In the K/DOQI classification, severe renal dysfunction corresponds to disease stages 4 and 5, with estimated glomerular filtration rates eGFR $<30 \mathrm{~mL} / \mathrm{min} / 1.73 \mathrm{~m}^{2}$ (or dialysis). In these terms, five-yr cumulative incidences of $10.9 \%$ and $14 \%$ were reported by Ojo et al. (5) and Lewis et al. (6) for severe renal dysfunction or ESRD.

It has long been known that serum creatinine, as a measure of kidney function, is inadequate by itself for early detection of chronic disease and for monitoring disease progression (7), and cyclosporineinduced disease is no exception in this respect (8). The MDRD equation is considered to afford adequate estimates of GFR for most kidney patients (3), but it is not clear that this is so for transplant patients (only one small study [9] has directly addressed this issue), and GFR appears to be no better than 1/SCr for prediction of all-cause mortality, cardiovascular mortality or kidney failure (10). In clinical practice and research (11), both serum creatinine and MDRD eGFR continue to be employed for evaluation of HT 
patients, and for the individual patient MDRD eGFR is indeed a simple function of SCr, age, sex and race. However, it is not clear to what extent the prevalences of K/DOQI-defined stages of chronic kidney disease, as diagnosed using MDRD eGFR, differ from the prevalences of degrees of renal dysfunction defined by conventional SCr cutoffs such as the $2.5 \mathrm{mg} / \mathrm{dL}$ used to define severe renal dysfunction by Taylor et al. (1) and others.

Furthermore, although numerous studies have reported that the incidence of severe kidney disease increases with time since transplantation $(1,5)$, there is relatively little information on the behavior of less advanced disease stages. In particular, the only previous examination of this issue in the authors' country was a single-center study of 262 patients (12).

In this article, we present the results of a multicenter cross-sectional study, CAPRI, in which, with the primary objective of determining the current prevalence of RD among Spanish HT patients, 1062 patients were graded for RD in accordance with both K/DOQI and conventional serum creatinine cutoffs so as to evaluate both the corresponding distributions among different degrees of RD and the changes in these distributions with time post HT.

\section{Methods}

\section{Participants}

Between October 2007 and March 2008, each of 14 Spanish heart transplant centers fed the CAPRI database with relevant data on all first HT patients aged $>18$ who presented during a pre-defined threemonth period for a routine follow-up examination more than $30 \mathrm{~d}$ post transplant (informed consent was a further condition that gave rise to no exclusions). Data were recorded for a total of 1062 patients $(82.5 \%$ males) with a mean age of $59.2 \pm 12.3$. Time since transplant ranged from one month to $22.3 \mathrm{yr}$ (mean $6.7 \mathrm{yr}, \mathrm{SD} 4.6 \mathrm{yr})$.

\section{Calculation of eGFR}

Glomerular filtration rate was estimated using the four-variable MDRD equation:

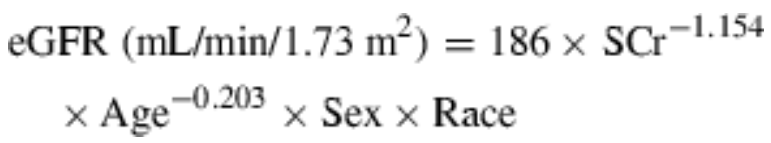

where $\mathrm{SCr}$ is in $\mathrm{mg} / \mathrm{dL}$, Age is in yr, Sex is 0.742 if female and 1 if male, and Race is 1.21 if black and 1 otherwise.

\section{Classification}

The degree of renal dysfunction of each patient at the time of the study was classified as absent-or-mild (AoM), moderate, or severe in accordance with two classifications: eGFR-RD, which used eGFR cutoffs of 30 and $60 \mathrm{~mL} / \mathrm{min} / 1.73 \mathrm{~m}^{2}$, and SCr-RD, which used SCr cutoffs of 1.6 and $2.5 \mathrm{mg} / \mathrm{dL}$ (Table 1). The eGFR cutoffs are those defining K/DOQI stage 3 chronic kidney disease, so that the eGFR-RD grade "severe" corresponds to K/DOQI stages 4 and 5, eGFR-RD "moderate" corresponds to K/DOQI stage 3, and eGFR-RD "AoM" includes K/DOQI stages 1 and 2 plus patients with no detected kidney dysfunction or damage. The SCr cutoff of $2.5 \mathrm{mg} / \mathrm{dL}$ separating SCr-RD "moderate" from SCr-RD "severe" is the same as used in the ISHLT Registry reports (1). The SCr cutoff of $1.6 \mathrm{mg} / \mathrm{dL}$ separating SCr-RD "moderate" from SCr-RD "AoM" has in recent years been used as a threshold, to exceed which is an indication for conversion to a less nephrotoxic immunosuppression regimen. 
Table 1. Degrees of renal dysfunction (RD) as defined by an eGFR-based classification (eGFR-RD) and a classification based exclusively on serum creatinine (SCr-RD)

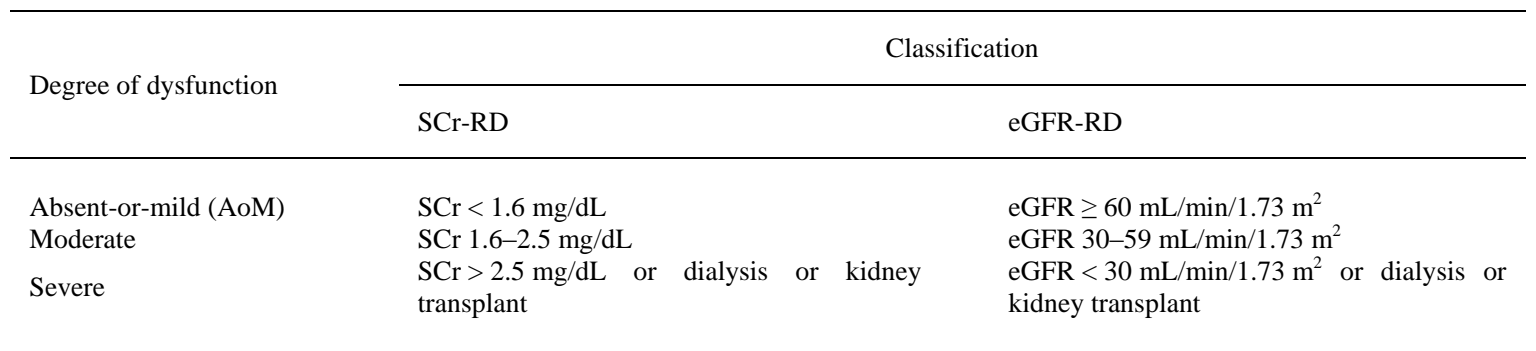

\section{Statistical analysis}

The prevalence of each degree of renal dysfunction in the study group was evaluated using both the eGFR-RD and SCr-RD classifications. To characterize the dependence of the distribution of renal dysfunction severity on time since transplant, prevalences were also evaluated in subgroups defined by the quartiles of the time-since-transplant distribution, Q1 $=2.70 \mathrm{yr}, \mathrm{Q} 2=6.17 \mathrm{yr}$ and Q3 $=9.54 \mathrm{yr}$.

\section{Results}

The exclusively creatinine-based classification SCr-RD classified $68.5 \%$ of all patients as having mild or no RD, $24.9 \%$ as having moderate RD, and $6.7 \%$ as having severe RD. The K/DOQI-based classification eGFR-RD classified $38.6 \%$ of patients as having mild or no RD, $52.2 \%$ as having moderate RD, and $9.2 \%$ as having severe RD. Of the 1011 patients who were neither on dialysis nor had undergone kidney transplantation, only $66 \%$ were assigned to the same RD category by both classification schemes, and the other 341 were all assigned to a more severe RD category by eGFR-RD than by SCr-RD: of the 721 patients assigned to the AoM category by SCr-RD, 314 (44\%) were identified by eGFR-RD as having moderate RD; and of the 263 assigned by SCr-RD to the moderate RD category, 27 (10\%) were identified by eGFR-RD as having severe RD.

Tables 2 and 3 present, for SCr-RD and eGFR-RD, respectively, the distributions among RD categories of patients with times since transplant in each of the four interquartile periods: $<2.7 \mathrm{yr}, 2.7-$ $6.2 \mathrm{yr}, 6.2-9.5 \mathrm{yr}$, and $>9.5 \mathrm{yr}$. According to SCr-RD, three quarters of patients with time since transplant shorter than $2.7 \mathrm{yr}$ have at most mild RD, and only 5\% severe RD. As time since transplant increases to $9.5 \mathrm{yr}$, there is a quite gradual increase in the proportions of both moderate and severe cases, but patients with time-since-transplant longer than $9.5 \mathrm{yr}$ show a more substantial change, the prevalence of severe $\mathrm{RD}$ rising to over $10 \%$ and that of moderate RD increasing by a third to over $32 \%$. Even so, even after $9.5 \mathrm{yr}$, most patients $(58 \%)$ are classified by SCr-RD as having at most mild RD.

Table 2. Prevalence of AoM, moderate and severe renal dysfunction (\%) among Spanish HT patients with times since transplant in each of the four interquartile periods, according to the classification SCr-RD (see Table 1)

\begin{tabular}{lcccc}
\hline & \multicolumn{3}{c}{ Time since transplant } \\
\cline { 2 - 5 } Degree of RD & $<2.7 \mathrm{yr}(\mathrm{n}=263)$ & $2.7-6.2 \mathrm{yr}(\mathrm{n}=267)$ & $6.2-9.5 \mathrm{yr}(\mathrm{n}=266)$ & \\
& & & 69.2 & 57.5 \\
Absent or mild (AoM) & 74.5 & 72.7 & 24.1 & 32.3 \\
Moderate & 20.5 & 22.5 & 6.8 & 10.2 \\
Severe & 4.9 & 4.9 & & \\
\hline
\end{tabular}


Table 3. Prevalence of AoM, moderate and severe renal dysfunction (\%) among Spanish HT patients with times since transplant in each of the four interquartile periods, according to the classification eGFR-RD (see Table 1)

\begin{tabular}{|c|c|c|c|c|}
\hline Degree of RD & $<2.7 \mathrm{yr}(\mathrm{n}=263)$ & $2.7-6.2 \mathrm{yr}(\mathrm{n}=267)$ & $6.2-9.5 \mathrm{yr}(\mathrm{n}=266)$ & $>9.5 \mathrm{yr}(\mathrm{n}=266)$ \\
\hline Absent or mild (AoM) & 47.5 & 37.5 & 37.6 & 32.0 \\
\hline Moderate & 46.8 & 55.8 & 53.8 & 52.3 \\
\hline
\end{tabular}

By contrast, according to eGFR-RD, even patients with time-since-transplant shorter than $2.7 \mathrm{yr}$ are most of them suffering from moderate-or-severe RD. The proportion with moderate RD is greatest among patients with time since transplant between 2.7 and $6.2 \mathrm{yr}$, among whom it rises by $9 \%$ with respect to the previous period at the expense of the AoM category. Among those with time-since-transplant between 6.2 and $9.5 \mathrm{yr}$, the severe RD category grows a little at the expense of the moderate RD category. Finally, almost $16 \%$ of patients surviving for at least $9.5 \mathrm{yr}$ have severe RD, over $52 \%$ moderate RD, and only $32 \%$ mild or no RD.

\section{Discussion}

The results of this study highlight the extent of the difference between grading HT patients for renal dysfunction using the K/DOQI system, with glomerular filtration rate estimated by the four-variable MDRD equation, and grading them using the serum creatinine cutoffs that have commonly been employed in heart transplantation programs. With moderate-or-severe RD defined as K/DOQI stage 3 or worse $\left(\mathrm{GFR}<60 \mathrm{~mL} / \mathrm{min} / 1.73 \mathrm{~m}^{2}\right.$ ), its prevalence among Spanish HT patients is over $60 \%$, almost twice the prevalence that results from using an SCr cutoff of $1.6 \mathrm{mg} / \mathrm{dL}$.

The inadequacy of SCr for grading renal function derives from three sources. In the first place, the creatinine filtration rate is not proportional to $\mathrm{SCr}$ but to $1 / \mathrm{SCr}$, which by itself would make the confidence interval for creatinine filtration given $\mathrm{SCr}$ quite wide at SCr levels below about $2.0 \mathrm{mg} / \mathrm{dL}$. Second, this source of uncertainty is amplified by tubular and extrarenal secretion of creatinine, which lower SCr (13), especially in patients with reduced GFR, in whom tubular secretion tends to increase to compensate for lost filtration (7). Third, there is intra- and interpersonal variation in these effects and in other determinants of SCr, such as diet and body habitus, and also uncertainty in SCr measurement. The final result is that an SCr level of $1.6 \mathrm{mg} / \mathrm{dL}$ can correspond to a GFR anywhere between about 20 and about $\left.80 \mathrm{~mL} / \mathrm{min} / 1.73 \mathrm{~m}^{2}\right)(7)$.

The four-variable MDRD equation estimates GFR, normalized to a body surface area (BSA) of $1.73 \mathrm{~m}^{2}$, as a function of $\mathrm{SCr}$, age, sex and race) (4). In effect, it uses the arguments other than $\mathrm{SCr}$, and a power of SCr slightly different from -1 , to account for some of the variation and bias in $1 / \mathrm{SCr}$ and urinary excretion as a result of the factors mentioned previously. It has not been validated among HT patients other than in a 27-patient study (9) that found it to overestimate GFR by $12 \%$; it is no better than 1/SCr for prediction of all-cause mortality, cardiovascular mortality or kidney failure (10); and in a study of 2095 white European adults (162 of them healthy) led to a misclassification rate of over $29 \%$ when used to grade patients in accordance with the K/DOQI stages of chronic kidney disease (14). However, it is the most reliable of all currently available means of estimating GFR without resort to biochemical analyses other than $\mathrm{SCr}$ (3), and among lung transplantation patients is a better pre-transplant predictor of its post-transplant decline than measured creatinine clearance (15).

The SCr cutoff used in this study to separate moderate and severe RD, $2.5 \mathrm{mg} / \mathrm{dL}$, was derived from the practice of the ISHLT Registry reports (1); and the cutoff used to separate AoM and moderate RD, $1.6 \mathrm{mg} / \mathrm{dL}$, was the threshold commonly used in recent years as an indication for a patient's conversion to a less nephrotoxic immunosuppression regimen. The corresponding eGFR cutoffs were the K/DOQI cutoffs separating stage 3 chronic kidney disease from stages 4 and 2, respectively. The K/DOQI cutoff between stages 2 and $3,60 \mathrm{~mL} / \mathrm{min} / 1.73 \mathrm{~m}^{2}$, was chosen because it is about half the normal adult GFR; and the cutoff between stages 3 and $4,30 \mathrm{~mL} / \mathrm{min} / 1.73 \mathrm{~m}^{2}$, because in light of NHANES III data, a lower GFR is only likely to be non-pathological for neonates (3). Although SCr and eGFR are not directly comparable (because of the influence of age, sex, race and BSA in eGFR), it seems clear from the results of this study that the $\mathrm{SCr}$ cutoff of $1.6 \mathrm{mg} / \mathrm{dL}$ must correspond to more advanced RD than 
$60 \mathrm{~mL} / \mathrm{min} / 1.73 \mathrm{~m}^{2}$. In adopting $1.6 \mathrm{mg} / \mathrm{dL}$ as a threshold indication for a change in immunosuppressive medication, HT physicians have accepted that a certain degree of RD is unavoidable or normal among HT patients and have only regarded RD as moderately severe, and hence indicative of a need to modify medication, when renal function has deteriorated to a relatively large extent. From a nephrologist's point of view, the absolute necessity of immunosuppression has, in effect, distorted the HT physician's perception of renal dysfunction. The discrepancy between SCr-RD and eGFR-RD prevalences observed in this study can be regarded as quantifying this distortion.

In the 1990s, several studies observed that a decline in renal function over the first 6-12 months postHT was followed by stabilization (16) or even by improvement (17) over the following 2-4 yr (18). Although the present study was cross-sectional, its results seem to bear out warnings that functional stability is unsustainable in the long run, because it is maintained by a progressively dwindling number of nephrons $(19,20)$. Whichever of the two RD classifications was used in this study, the prevalence of severe RD increased at an accelerating rate with time since transplant, the greatest difference being seen between patient groups surviving less than or longer than $9.5 \mathrm{yr}$. Although this time dependence may have been partly as a result of advances in management, patients with long survival times having in many cases been subjected in their early post-HT yr to more nephrotoxic regimens than more recent patients, the fact that the moderate and severe RD groups are also larger for 2.7-6.2 yr than for sub-2.7-yr survival suggests that this is not the sole cause, and that even with current regimens the risk of nephropathy increases with time.

It is worth pointing out that the analysis of prevalences among patients with different survival times also shows that, although the disagreement between SCr-RD and eGFR-RD is greatest between 2.7 and $9.5 \mathrm{yr}$ post-HT, it is already very considerable among patients with times since transplant shorter than 2.7 yr. Over a quarter of these patients are graded as having AoM RD by SCr-RD, but more severe dysfunction by eGFR-RD. Since remedial or damage-limiting measures, if feasible, are presumably more effective the earlier they are initiated, the greater sensitivity of the K/DOQI-based classification is potentially of particular value for these patients.

The major limitation of this study is its cross-sectional nature. As our data do not allow Kaplan-Meier estimation of the intrinsic risk of developing renal dysfunction, our results are not directly comparable with the ISHLT Registry data cited in the introduction. Nor can we report the "actual" cumulative incidence of severe or moderate-or-severe RD. A further limitation is that the MDRD equation has not been validated for HT patients and that its accuracy in the present study was not checked by determining GFR with a reference method (measurement of the clearance of an exogenous marker such as inulin, ${ }^{51} \mathrm{Cr}$ EDTA, ${ }^{99 \mathrm{~m}}$ Tc DPTA, or ${ }^{125}$ I-iothalamate) (21), which is not feasible in routine clinical practice; it is this uncertainty that has prevented our drawing blunter conclusions as to the utility of SCr. However, if the most likely source of MDRD error for HT patients is their tendency to low muscle mass, then the observed differences between SCr-RD and eGFR-RD prevalences are no exaggeration, because this tendency would tend to make eGFR overestimate GFR, and so reduce the difference between SCr-RD and the K/DOQI-based prevalences.

In conclusion, the prevalence of severe RD increases with time since transplant. Assuming that for HT patients it is appropriate to grade renal dysfunction using the K/DOQI chronic kidney disease stages, with estimation of GFR by the four-variable MDRD equation, then (i) direct SCr-based grading of HT patients should be abandoned as excessively insensitive, and (ii) the need for less nephrotoxic immunosuppressive regimens and other renoprotective measures is of greater magnitude than is suggested by SCr-based grading.

\section{Acknowledgements}

We are grateful to the researchers and staff of all the Spanish heart transplant centers that contributed data to this study, to Soly Santiago for the statistical analyses, and to Ian-Charles Coleman for helpful criticism and for the English version of this paper.

\section{Funding sources}

Financial support of this research by an unrestricted grant from Astellas Pharma and the Spanish Ministry of Health and Consumer Affairs through the Carlos III Institute Cardiovascular Research Networks RECAVA (of which MC-L, MP, BDM, LF, and JM are investigators) and REDINSCOR (of which JD, LA, ER, LA-P, and IG are investigators). Otherwise, all the authors declare that they have no financial relationships to disclose. 


\title{
Author disclosure
}

The authors also declare that the present manuscript has not been published previously and that it is not under consideration for publication elsewhere, except that the Abstract was presented at the International Society for Heart and Lung Transplantation 29th Annual Meeting and Scientific Sessions, Paris (France) April 22-25th, 2009.

\begin{abstract}
Appendix
The following investigators also contribute to CAPRI study: Zulaika Grille, Carmen Naya and Victoria Pardo (Complejo Hospitalario Universitario A Coruña, La Coruña); Francisco González-Vilchez; Tamara García-Camarero; Miguel Llano (Hospital Universitario Marqués de Valdecilla, Santander); Miguel Angel Gómez Sánchez; Nuria Ochoa; Marta Paradina; Pilar Escribano (Hospital Universitario 12 de Octubre, Madrid); Jesús Palomo Álvarez; David Pascual Hernández (Hospital General Universitario Gregorio Marañón, Madrid); Luis Martínez-Dolz (Hospital Universitario La Fé, Valencia); José Luis Rodríguez Lambert (Hospital Universitario Central de Asturias, Oviedo); Félix Pérez Villa (Hospital Clinic i Provincial, Barcelona); Juan Carlos Castillo Domínguez; Amador López Granados (Hospital Universitario Reina Sofía, Córdoba); Javier Segovia; Manuel Gómez Bueno (Clínica Puerta de Hierro, Madrid); Domingo A. Pascual Final (Hospital Universitario Virgen de la Arrixaca, Murcia); Teresa Blasco Peiró (Hospital Universitario Miguel Servet, Zaragoza); Javier López Díaz (Hospital Clínico Universitario, Valladolid); Vicens Brossa Loidi (Hospital de la Santa Creu i Sant Pau, Barcelona).
\end{abstract}

\section{References}

1. Taylor DO, Edwards LB, Aurora P et al. Registry of the International Society for Heart and Lung Transplantation: twenty-fifth official adult heart transplant report - 2008. J Heart Lung Transplant 2008: 27: 943. Data available at http://www.ishlt.org/registries/.

2 Myers BD, Ross J, Newton L, Luetscher J, Perlroth M. Cyclosporine-associated chronic nephropathy. N Engl J Med 1984: 311: 699.

3 National Kidney Foundation Kidney Disease Outcome Quality Initiative Advisory Board. K/DOQI clinical practice guidelines for chronic kidney disease: evaluation, classification, and stratification. Am J Kidney Dis 2002: 39(Suppl 2): S1.

4 Levey AS, Greene T, Kusek JW, Beck GJ, MDRD Study Group. A simplified equation to predict glomerular filtration rate from serum creatinine. J Am Soc Nephrol 2000;11:155A (abstract).

5 Ojo AO, Held PJ, Port FK et al. Chronic renal failure after transplantation of a nonrenal organ. N Engl J Med 2003: 349: 931.

6 Lewis GD, Frankel DS, Brown RN et al. Predictors of early development of chronic kidney disease following heart transplantation. J Heart Lung Transplant 2007: 26: S137.

7 Shemesh O, Golbetz H, Kriss JP, Myers BD. Limitations of creatinine as a filtration marker in glomerulopathic patients. Kidney Int 1985: 28: 830.

8 Golbetz H, Perlroth M, Stinson E, Myers BD. Limitations of creatinine in quantifying the severity of cyclosporineinduced chronic nephropathy. Am J Kidney Dis 1986: 8: 332.

9 Delanaye P, Nellessen E, Grosch S et al. Creatinine-based formulae for the estimation of glomerular filtration rate in heart transplant recipients. Clin Transplant 2006: 20: 596.

10 Menon V, Shlipak MG, Wang X et al. Cystatin C as a risk factor for outcomes in chronic kidney disease. Ann Intern Med 2007: 147: 19

11 Shiba N, Chan MC, Kwok BW, Valantine HA, Robbins RC, Hunt SA. Analysis of survivors more than 10 years after heart transplantation in the cyclosporine era: Stanford experience. J Heart Lung Transplant 2004: 23: 155.

12 Garrido IP, Crespo-Leiro MG, Paniagua MJ et al. Independent predictors of renal dysfunction after heart transplantation in patients with normal pretransplant renal function. J Heart Lung Transplant 2005: 24: 1226.

13 Levey AS. Measurement of renal function in chronic renal disease. Kidney Int 1990: 38: 167.

14 Froissart M, Rossert J, Jacquot C, Paillard M, Houillier P. Predictive performance of the Modification of Diet in Renal Disease and Cockcroft-Gault equations for estimating renal function. J Am Soc Nephrol 2005: 16: 763.

15 Al-Naamani N, Maarouf OH, Wilt JS et al. The Modification of diet in renal disease (MDRD) and the prediction of kidney outcomes after lung transplantation. J Heart Lung Transplant 2008: 27: 1191.

16 Gonwa TA, Mai ML, Pilcher JB et al. Stability of long-term renal function in heart transplant patients treated with induction therapy and low-dose cyclosporine. J Heart Lung Transplant 1992: 11: 926.

17 Ruggenenti P, Perico N, Amuchastegui CS, Ferrazzi P, Mamprin F, Remuzzi G. Following an initial decline, glomerular filtration rate stabilizes in heart transplant patients on chronic cyclosporine. Am J Kidney Dis 1994: 24: 549.

18 Van BurenDH, Burke JF, Lewis RM. Renal function in patients receiving long-term cyclosporine therapy. J Am Soc Nephrol 1994: 4: S17.

19 Myers BD, Newton L. Cyclosporine-induced chronic nephropathy: an obliterative microvascular renal injury. J Am Soc Nephrol 1991: 2: S45. 
20 Wilkinson AH, Cohen DJ. Renal failure in the recipients of nonrenal solid organ transplants. J Am Soc Nephrol 1999: 10: 1136.

21 Mariat C, Alamartine E, Barthelemy J-C et al. Assessing renal graft function in clinical trials: can tests predicting glomerular filtration rate substitute for a reference method? Kidney Int 2004: 65: 289. 